\title{
Review Study on Smart Nanomaterials and their Applications
}

\author{
Ankit Kumar Mishra ${ }^{1}$, Janani KaviPriya V.S ${ }^{1}$, Eldho Jose ${ }^{2}$, Anjali Mohan ${ }^{2}$, Masetty Akshay ${ }^{3}$ \\ 1Department of Research and Development, Abyom Space tech and Defence Pvt. Ltd, Gorahkpur, India \\ 2Department of Chemical Engineering, National Institute of Technology, Kozhikode, India \\ 3Department of Mechanical Engineering, Anurag University, Telangana, India \\ *Corresponding author Email: ankitkumarm1998@gmail.com
}

\begin{abstract}
Smart nano materials are becoming increasingly popular in several fields of research. Some of the reasons for a lot of study in this field include the unique properties of these materials to adapt and respond to their environment, as well as increased qualities when combined with other materials. We have highlighted some of the most interesting nanomaterials in this review paper, including carbon-based nanomaterials such as nanodiamonds, metal-based nanoparticles such like gold, silver, and aluminium nanoparticles, and electrospun nanofibers. The characteristics and uses of these chosen materials were described along the review. In addition, some of the most notable characteristics or qualities of each material are addressed, as well as their purposes. This collaborative research will aid in a briefing on the key characteristics of certain frequently utilized nanoparticles as well as their application areas.

Keywords: Nanomaterials, Composites, Nanofibers, Nanodiamonds, Parameters.
\end{abstract}

\section{Introduction}

Smart nanomaterials are also known as intelligent materials are gaining importance due to their versatile and unique features. They provide a solid basis for a variety of applications. Many smart nanomaterials have been produced and successfully employed in many applications during the years of progress in the field of nanotechnology; they are typically called 2nd or 3rd generation nanotechnology applications [3]. The smart materials at the beginning were regarded as materials that respond to the environmental factor promptly, but now these are defined as the materials that respond to stimuli that result in a reversible change in their functional properties [2]. The stimuli are defined as the change in environmental factors such as changes in temperature, electricity, magnetic field, stress, pressure, etc... All these will provide necessary functions in the smart nanomaterials to create potential applications. It is still difficult to fabricate smart nanomaterials but they are still widely considered due to their manifold functions, self-sensing, and self-adaptability which make them unique [1]. They found applications in Biomedical, Energy Devices, Healthcare, Security and Defense, Environmental Engineering but there is a significant increase in the use of smart nanomaterials in the aerospace industry. Smart nanoparticles are getting a lot of traction in the aircraft business because the applications are exposed to a lot of changes because they are put in an open environment. As a result, smart nanomaterials are gaining a lot of traction in the aerospace industry [1]. Different nanomaterials have been developed over the years which could be used as smart materials that show intellectual performance during changes produced in the environment such as metallic nanomaterials, carbon nanomaterials, and electrospun nanofibers. The metallic nanomaterials such as gold nanoparticles show good catalytic properties; hence they are used in the manufacturing of fuel cells. The silver nanoparticles exhibit various colors in the spectrum when it absorbs light hence, they are used to build sensing devices that can monitor the performance of the structure, and aluminium nanoparticles exhibit lubricative property which reduces friction in between the parts of aircraft. The Carbon nanomaterials such as graphene nanocomposites, MWCNT nanocomposites, and Nanodiamond Composites exhibit decent mechanical properties. MWCNT has a high tensile strength hence it is used as a strain sensor. Nanodiamond composites have a high melting point and exhibit high crystalline property thus they are used to protect sensors in aircraft. The Graphene nanocomposites show decent electrical and thermal conductivity therefore they are used as filler in sensor and solar cells. Electro spun nanofibers have been developed as 2D and 3D nanomaterials. Allowing them to be used in a variety of aerospace applications. They're utilized to make things like monitoring systems, sensors, sound absorbers, filters, and fuel cells, among other things. The article describes the various smart nanomaterials developed over the years their properties and applications. Al Arsh Basheer (2020) the very unique property of smart nanomaterials to adapt to changes in the environment has attracted the aerospace industry. The unique properties of these materials include response to temperature, radiation shielding capacity, structural integrity etc. The study emphasises on the classification of smart materials and explains the working of the same which basically is by response or change in its properties to different environments. The different types of smart materials are explained and thermally responsive materials like shape memory alloys (SMA) are given importance. The SMA used in aircraft wings can change its shape when the wing speed are varied thus reducing its structural damage. Other aerospace applications include reducing vibrations in aircraft wings, peizoelectric materials are used for making sensors etc. The aerospace industry today faces many challenges and nano-smart materials are considered as a great asset for this industry [1]. P. Lakshmi et al. (2015) In aerospace engineering it is very important to do periodical maintenance on structures. Using sensitive materials provides the real-time information about the material itself. In SHM the 
sensory material is embedded in the material itself as a filler. Glass fiber reinforced polymers (GFRP) are having good mechanical properties and can be used as the material for the aircraft parts. The CNF fibers are used as sensory fiber due to its high moduli. PVA-CNF fibers are incorporated with the GFRP material for the best responsive system. The electrospun nanofibers are mixed with the polymer solution and then incorporated to the GFRP sheets. In addition to that Tensile test was performed and it was seen that the as the level of stress is increased the damage increase and the sensor has no role in the strength of the material. The electrical conductivity test showed that the nanosensor addition has increased the conductivity of the material thus increasing its sensitivity. So, the use of this CNF based senors has given a new beginning in the area of SHM [4].

J.R Potts et al. (2011), mentioned about new breakthroughs in polymer nanocomposites have changed nanoparticle research. This research looks into graphite nanoplatelet fillers made from graphite intercalation compounds (GICs) and fillers made from graphite oxide (GO). The mechanical and thermal methods used for the exfoliation of graphite are examined the enhanced properties of these derived fillers are also discussed. The uses of GO derived fillers are also given importance due to the increased oxygen-based functionalization. The dispersion of these derived fillers in the polymers to make composites by non covalent and covalent dispersion methods are studied. The morphological and crystallization behaviour of the platelets by TEM and WAXS shows that the exfoliated platelets have more aspect ratio compared to the intercalation compounds which is favourable for better mechanical, thermal and electrical properties. Other properties like rheological, visoelastic, conductivity etc are also studied. The use of GNP and GO derived fillers is relatively cheap since it is derived from graphite and can be used as an alternative to CNT nanocomposites which is having wide applications in many areas [5]. Z.Li et al (2016) Galatic cosmic rays mainly consist of high energy proton radiations and these radiations can adversely affect the material structure of the spacecraft and also cause genetic changes in human cells. This study focuses on PMMA/MWCNT based nanocomposite that can be used as a shielding against these ionising radiations. It was seen that low charge to mass ratio materials has better shielding capacity. Aluminium alloys have good shielding property but it is heavy so using poly methyl methacrylate (PMMA) is considered as an effective material due to the abundance of hydrogen and also light weight. The formation of secondary radiations is resolved by using MWCNT as a filler to the polymer because of its property of minimizing structural damage on exposing to these radiations. The fabrication of the composite is done by melt mixing, moreover the dispersion quality is increased by functionalising the MWCNT using carboxyl group. To gain an understanding of proton transmission and secondary neutron production, WETs and WEADs are used. And it was found that the composites are more effective compared to aluminium and the neat polymer furthermore, the composites also showed better tensile as well as thermal properties. The proton test done on the composite also showed that the air trapped inside the material during fabrication disappeared due to the ionizing radiation [6]. V.Mohan et al. (2018) The graphene-based nanocomposites is a major area of research due to its wide range of applications. This study is based on different type of graphene polymer composites their synthesis functionalization characterization and major applications. Graphene synthesised by chemical vapour deposition (CVD) are used in coating, electronics applications whereas liquid phase exfoliatied graphene are used in composites energy storage applications. The graphene metal/ceramic composites showed ageing resistance and other superior mechanical properties. The major applications of these composites are in sensors, automobile components, membranes etc. The study also emphasis on developing a lightweight structural material which can operate in low temperature conditions. Carbon fiber reinforced polymer (CFPR) can replace metal cryogenic fuel tank for better life of the space launch vehicles and also reducing cost. Some carbon based nanofillers can be used to strengthen the properties of the CFPR which can be brittle on low temperature environments due to the thermosetting resins used as its matrix. Thus, structural improvements in graphene-based materials can make remarkable changes in many areas of science [7]. V.Mochalin et al. (2015) There are different types of carbon-based polymer composites that has been under research for several years. Due to the high ratio of nanoparticles, the interphase volume will be large, and the composite properties will be equivalent to nanofillers. Nanodiamonds are unusual among carbon-based fillers because of their spherical shape, which allows them to spread widely in the medium. Furthermore, the core is inert, while the exterior surface is reactive owing to the inclusion of functional groups. Because of their zero-dimensional structure. In comparison to CNT and graphene-based fillers, the tiny size also lowers manufacturing costs. The epoxy ND composite is also an important material since it is used as a matrix for CFR composites in aerospace applications. The studies showed that high concentration of ND made the material hard whereas low loadings increased the mechanical properties. Thus, advancements in the nanodiamod composites are a promising area of research as it has clear advantage over CNT and other nanomaterials [8].

A.Kausar (2017) Nano diamond polymer composites are regarded as a promising material in the area of composite. The study focuses particularly on the significance of nanodiamond epoxy composites. NDs generally have better properties than its constituents because it exhibits the characteristics of diamonds as well as that of nanoparticles. The major synthesis of NDs is by detonation of carbon material which produces particles of very small size. It was also seen that with different concentrations of NDs different properties of the composites are enhanced. The epoxy nanocomposites are having range of applications including aerospace. ND reinforcement in epoxy composites showed great increase in glass transition temperature along with mechanical and electrical properties. The major applications of these composites are in electrical devices due to electromagnetic compatibility, shielding materials etc. The property of ND to create very closely spaced particles in the interphase at low loadings thus providing better thermal and mechanical strength makes it a very unique nanoparticle compared to its other constituents [9]. R Jose Varghese et al (2019) Nanomaterials are particles with nanoscale dimension where surface and interface properties dominate over bulk properties. Nanomaterials could be classified under ultra-fine particles due to their nanoscale dimension. Synthesis of nanomaterials can be done using different techniques 
which includes mechanical milling which has the basic principle of energy transfer to sample from the tungsten or steel balls present. Electrospray method is similar but slower. Laser ablation is another method which uses laser rays penetration depending on the wavelength of the rays and material refractive index. Other techniques would be plasma arcing, reverse micelle method etc. All these methods of production of nanomaterials are beneficial to the development but at the same time their environmental impacts should be given more emphasis which helps in choosing a process both efficient and ecofriendly [10]. Novikov et al (2017) the durability is an important factor regarding a spacecraft. The nanomaterials with their unique characteristics like high mechanical, thermal and optical properties promise a higher durability spacecraft. As far as a spacecraft is concerned radiation shielding is a necessity. Nanomaterials with large grain boundaries exhibit improved radiation shielding. They not only reduce the spacecraft's total weight, but they also improve its operation and power generation. The use of CNTs can increase the tolerance against structural deformities. Overall, the nanomaterials do an exceptional role in enhancing the durability and strength of spacecraft promising a brighter future for the same [11].

Z. H. Khan (2016) The paper provides an insight to the various classification, types and applications of nanomaterials. Nanomaterials due to their nanoscale dimension exhibit exceptional properties that could present $\mathrm{n}$ number of possibilities. As a result of their GMR, they display specific features such as melting point depression, i.e., a lower melting temperature relative to bulk material, which enhances storage capacity. The paper details the types of nanomaterials that include CNTs, that stand out with its strength and light weightiness than steel and also points at its high thermal and electrical conductivity, nanodiamonds, used for hard coatings and optical window, graphene, surpasses copper with its high mobility and current densities, $\mathrm{ZnO}$, used in paints and finally fullerene, the zero-dimensional form of carbon. To brief it nanomaterial applications, range from agricultural science to space technology covering optical, electronics, fuel cells, hydrogen storage, medicine etc. But the toxicity of nanomaterials again due to their small size should be precluded by close monitoring [12]. Harish kumar et al (2018) Metal nanoparticles, a Faraday discovery, are an essential field of nanotechnology due to their exceptional properties optically, thermally, mechanically and electrically. Their thermal properties can be used for electronic wiring and their electrical characteristics for superconductivity materials. In addition to these they serve as excellent catalysts especially gold and silver nanoparticles which can be purposeful in fuel cells. The both photolytically and radiolytically synthesised particles lay forward infinite possibilities in various fields. But the disadvantages they posses like the particle instability, impurities building up, coagulation etc should be taken care of for better manipulation of these particles [13]. David Thompson (2007) The gold nanoparticles has attracted many researchers due to their high activity even under mild conditions. These highly active nanoparticles exhibit excellent catalytic and lubricant properties. Their catalytic property can be used in the removal of carbon monoxide by oxidation thus controlling pollution and emission. Not just carbon monoxide they also detect harmful nitrogen compounds and hence helps to purify air. As we know fuel cells are excellent source of electric power in spacecrafts. The catalytic property of nano gold can be manipulated in fuel cells. Gold nanoparticles are easily available which makes them cost efficient and they also exhibit great stability. They can also be used in sensors to detect poisonous and inflammable gases. The gold nanoparticles if manipulated properly can be highly beneficial commercially in lots of fields including medical due their properties [14]. Di Yan (2020) Nanoscale aluminium exhibit improved characteristics than its bulk material like reactivity, specific impulse, density and energy releasing. Due to these properties' addition of nano aluminium to liquid propellants enhances the pay load of rockets and missiles by enhancing the energy performance. The system for testing the same was designed with aviation kerosene and solid fuel as nano aluminium. The system was designed aiming for a better accuracy, efficiency, light weight and low energy consumption which was obtained at the end of the experiment. But the proper mixing of the fuels posed a challenge. Further optimisation can help overcome this challenge and help in designing a highly efficient fuel pump [15]. Prasad Govindrao Jamkhande (2019) the nanotechnology is an emerging field. The application of nanomaterials is immense due to their exceptional properties which arise due to their nanoscale dimension. The synthesis of these particles in a befitting manner is highly essential. This paper discusses the various synthesising methods of nanoscale particles along with their advantages, disadvantages and applications. New methods or improvising the existing methods for the same is regarded important [16]. Wee-Eong Teo (2016) Electro Spun Nanofibers are being used in the different aspects of the aerospace industry for its development. The electrospun nanofibers when placed between composite laminates will increase their fractural properties, modulus of rupture, impact energy. Nanofibers with finer diameters in the composite have a higher modulus of rupture than the larger diameters and Nanofibers with the smallest thickness have high flexural strength and Modulus of Elasticity than thick layers, but the thicker layers provide better arrest crack propagation. Due to the above properties and its lightweight, the electrospun nanofibers are used in the micro-air-wing (MAW) skin. Thus, the electrospun nanofibers are used to improve the structural enhancement, noise control, and maintenance of aircraft and military vehicles used in the aerospace industry [17].M.S. Nisha et al (2016) Nanofibers have started to play a major role in the aerospace industry, the nanofibers are used to manufacture structural health monitoring systems, and due to this system, we can constantly monitor the health of the aircraft and its aging. Thus, we can able to reduce Maintainance costs, minimizes periodic inspection. The PVDF-MWCNT fibers embedded with GFRP are used to manufacture structural health monitoring of composite structures. The PVDF-MWNCT is used in composite to sense the damages, vibrations, eddy current damping, lamb waves. The PVDF-MWCNT is prepared by the electron spinning process. Mechanical Tests are conducted to find out the tensile behavior of the PVDF nanofiber, The tests applied are the Monotonic tensile test, Incremental tensile loading, the tests are applied till the specimen fracture. At last, by an electronic microscopic we determine the phase and crystalline structure of PVDF-MWCNT fiber [18].

M. Badmus et al. (2020) The nanofibers synthesized by using electron spinning procedure have weak strength which doesn't allow them to be used for many applications. To improve their properties hierarchical morphology is used which 
introduces additional structures on electrospun nanofibers. By controlling electrospinning parameters, we can improve the properties but also eliminate the undesirable properties of the electro spun nanofibers. Hierarchical electro spun nanofibers provide a high-porous mesh structure that could be used for energy storage and also increase the multifunctionality of the nanofibers. There are two types of hierarchy structures primary and secondary structures. The primarily structured fibers are fabricated using the electron spinning process with specific feed requirements and by using triaxial spinnerets. Different morphologies can be obtained in the primary structure such as honeycomb, core-shell fiber, block copolymers, bead-on-astring, and side-by-side fibers. Among all of them, the honeycomb structure is very famous because of it's self-assembly nature. The primarily structured nanofibers are mostly used in catalysis and filtration applications [19]. Michalel T Barako et al (2018) The Nanomaterials have been playing a crucial role in the development of thermal management to create highpowered electronics which are used in aerospace platforms. Nanomaterials are used to construct advanced thermal management architecture; the nanomaterials are used to remove the thermal stresses and improve the performance limitations of the electronics used in aerospace. The aerospace industry uses high powered electronic systems which have to manage extreme thermal loads due to the generation of heat fluxes which in return causes limitations in the performance of the device, this is where nanomaterials play a crucial role, their rational design can improve the thermal storage, thermal conduction, tailor radiated surfaces, and multi-phase heat transfer. The Integrated nanomaterials are co-designed into a new thermal-electrical architecture and the integration at the multiple length scales causes complete elimination of heat resistance pathways. Due to this, we can avoid system failures during a launch [20]. D. Mailly (2009) There are many techniques present to manufacture objects on the scale of $100 \mathrm{~nm}$, but one of the main and efficient techniques is lithography, the various processes under the lithography are Ion Beam lithography technique, Electron Beam Lithography technique, Optical Lithography technique. These processes are classified based on radiation used: In the case of optical lithography, we use photons, electrons in the electron beam, and ions in ion beam lithography. The steps involved in the lithography process are, at first, to prepare our desired pattern or structure we take a material called substrate; it is flat shaped material (ex: Silicon Wafer) now we spread a layer of energy sensitive material called resist. Now we expose it to radiation at some well-defined spots to create a chemical change, these steps define the size of the pattern. Now we remove the resist from exposed regions in case of positive resist, but if it is negative resist, we remove the unexposed region. Then after many other techniques can be applied for the transfer of resist onto a substrate such as etching, electroplating, or lift-off. This entire process is called Lithography and is the most widely used technique in the case of large volume printing.[21]

\section{Discussions}

Gold Nanoparticles: Catalytic property Gold nanoparticles, making them excellent catalysts. These catalysts work in even the most inhospitable environments. In the chemical process of ethylene glycol and methanol to produce methyl glycolate the nano gold catalysts exhibited enhanced stability. The methyl glycolate so produced is used in semiconductors and cosmetics. The nano catalysts served greatly important in VAM manufacture by greatly increasing the selectivity and activity of the process. During 110 days, the average activity of glucose to gluconic acid conversion in alkaline aqueous solution was $140 \mathrm{mmol}$ min-1 gAu-1. They help in pollution control by the oxidation of carbon monoxide and other nitrogen containing compounds in atmosphere. The catalysts exhibit a great role in fuel cells due to its CO oxidation. The graph shows that the $\mathrm{Au} / \mathrm{TiO} 2$ catalyst has a good selectivity for $\mathrm{CO}$ oxidation and works at a low temperature. It outperforms the $\mathrm{CO}$ tolerant PtRu and PtMo technologies. As a result, a highly efficient eco-friendly fuel cell is on the horizon. [14].

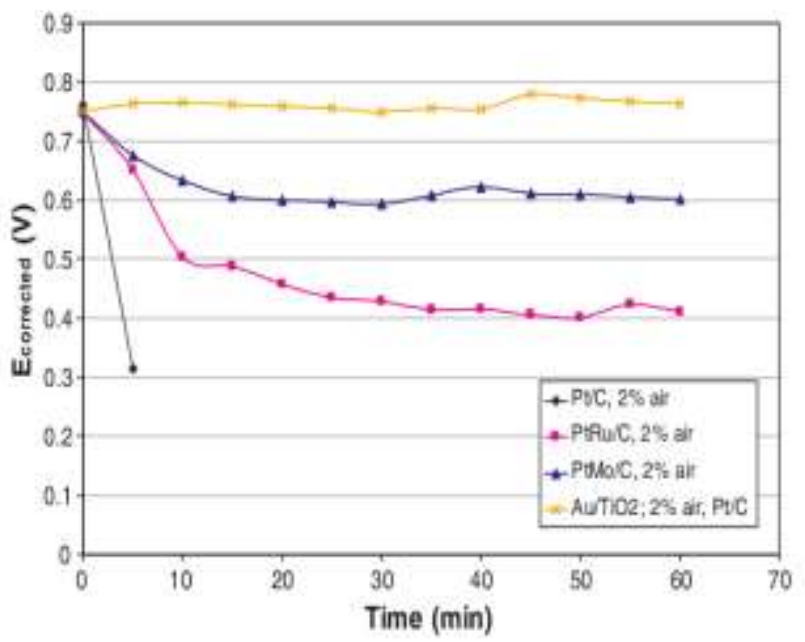

FIGURE 1. comparison of CO tolerance [14].

Optical Functionality: The gold nanoparticles were observed to round in shape with distinguishable features through the transmission electron microscopy. The height analysis showed around 18nm. The ruby colour of the colloidal solution observed indicates optical spectra. The color of the glass covered with Au nanoparticle showed light pink with a maximum 
wavelength of $521 \mathrm{~nm}$. This color remained the same irrespective of time and position indicating the stability of gold nanoparticle.
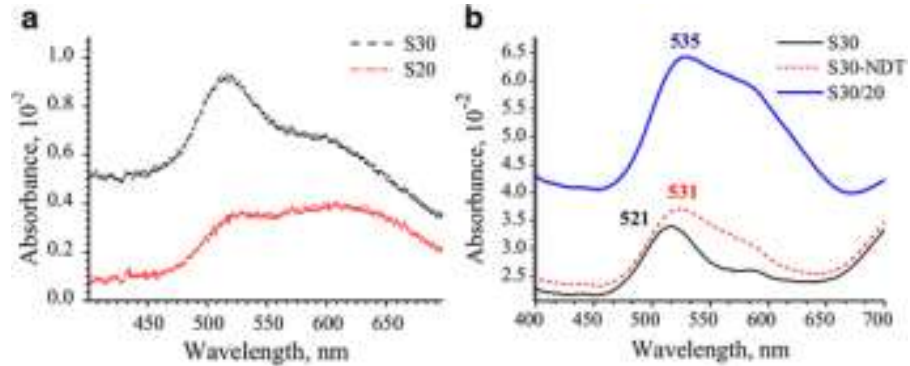

FIGURE 2. Optical spectra with characteristic LSPR band of Au particles [22]

Coupling at larger wavelength in the range of 550-600nm formation of band occurs. It was observed a shift of Plasmon resonance in blue and red wavelength with the morphology of the nanoparticle. Also, the optical activity of nanoparticle depended on the angle of incidence of the light, size, shape and mutual arrangement of the particles. This plays an important role in image sensing [22]. Increased Raman scattering and electromagnetic is highly useful in cancer therapies. The Mie scattering of these particles again emphasizes its medical importance. The light scattering of gold nanoparticles in inexpensive compared to MRI [23].

\section{Silver nanoparticle}

UV-V is light spectroscopy: The silver nanoparticle has got an appreciable aspect ratio. This along with the size affects the absorbed light wavelength. The particles exhibit various colours depending on their size and shape. The conduction of electrons and mutual interactions among the particles affect the vibrant colours these particle showcase. They exhibit rainbow colours. These properties highly benefit in the treatment of cancer, photovoltaic devices and sensing devices [24]. The UV- Vis spectroscopy indicated the low absorption of longer wavelengths. Shorter wavelengths on the other hand increased the absorption. A steady decrease in UV region is also observed [25].

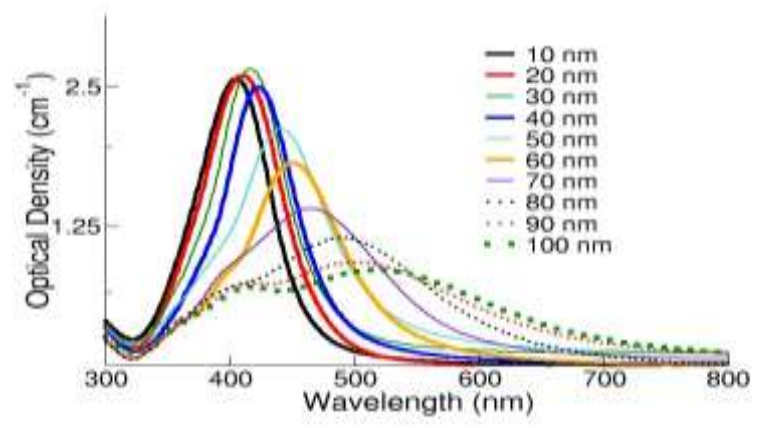

FIGURE 3. Optical absorption spectra of Ag NPs [25]

Plasmon resonance: Optical characteristics of silver nanoparticles in the visible and near infrared ranges have been discovered. This is evidenced by the high peaks in their spectral extinction. The Mie theory emphasises the influence of particle size in this. The SPR is more prominent with flat and discoid shapes [24]. The TEM images produced various shaped distinguished by the various colours they owned. A triangular one had red colour and a pentagon had a green colour [26]. The LSPR produces strong electromagnetic field on the particle surface due to its high molar extinction coefficient and Rayleigh scattering. This also resulted in prominent Raman scattering [24]. These properties come handy in photonics and biological applications [24].

\section{Aluminium Nanoparticle}

Lubricative Property: The SEM image revealed A12O3 agglomerates. This nano oil had increased viscosity and VI value compared to the diesel oil normally used. The thermal stability increased and flash point temperature was decreased [27]. The SEM images revealed the direct dependence of dispersion on Zeta potential. The dispersion can also be related to optical absorbance spectrum which gives the stability of nano aluminium oil. Al2O3 nanoparticles are hard and heat and wear resistant. The four-ball test confirms the same. The friction coefficient gave the energy consumption is because to the friction, wear scar diameter and the wear rate. The test conducted at different $\mathrm{Al} 2 \mathrm{O} 3$ concentrations gave satisfying results at $0.1 \mathrm{wt} \%$. At this concentration excellent wear rate and reducing friction is attained [28]. This property can be manipulated for reducing friction between different parts in an aircraft [28]. 

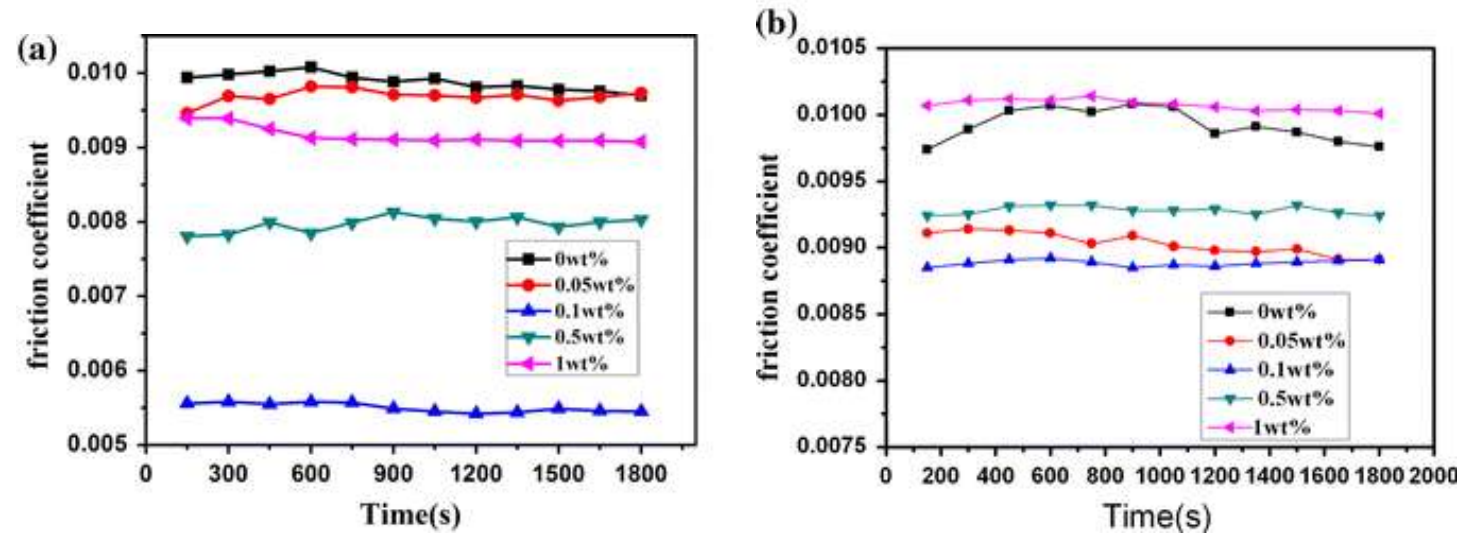

FIGURE 4. Curves illustrating four-ball friction coefficients for various $\mathrm{Al} 2 \mathrm{O} 3$ concentrations over time [27].

Optical property: Micrographs showed the dependence of coatings on grain size. Larger the coating bigger the grain size. The particle depends on the orientation and rotation. The come together to form dimers and trimers. The peak position shifted with thickness. Formation of dimers resulted in enhanced peak. Theoretical calculation pointed at the plasmon resonance in the UV region. The larger sizes exhibited broader absorption in NIR region [29]. The LSPR of Al nanoparticles showed a broad absorption at a $350 \mathrm{~nm}$ wavelength. This property of nanoparticles depends on the mass thickness and temperature of the particles and can be manipulated in increasing the solar cell functionalities [30].

\section{Nanodiamond Composites}

Nanodiamonds are zero-dimensional allotrope of carbon that are having very unique and superior properties due to its small size and high dispersion with the matrix. There are lot of areas of advancements in this area and it is supposed to replace most of the carbon-based filler used currently. Because of their excellent mechanical, thermal, and electrical characteristics, nanodiamond composites have a wide variety of applications. The major synthesis of nanodiamonds is by detonation. Some of the major properties and applications of nanodiamonds are discussed below [8, 34].

Microstructure: It was discovered that the microstructure of NDs has a more S/V ratio, which is responsible for the high interphase thickness. Moreover, they form agglomerates or clusters of nanoscales. The unique structure also helps in the attachment of many functional groups thus the material can be modified with desired properties. It is also showing very high dispersive property which is responsible for good structural materials. Due to its spherical nature the NDs will not increase the viscosity of polymer which aids in the formation of good quality composites compare to other filler [8, 34].

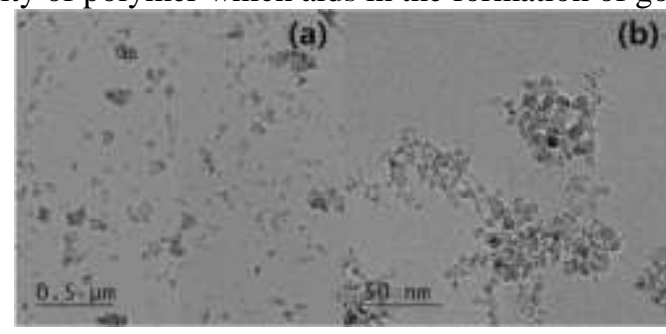

FIGURE 6. Functional groups attached to ND surface [8]

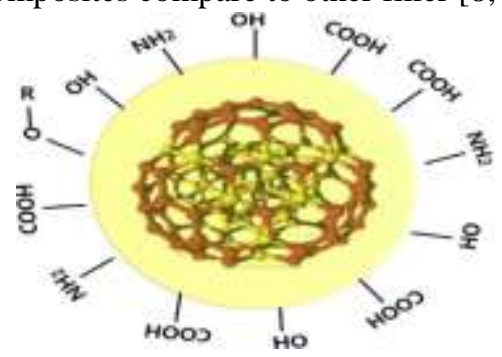

FIGURE 5. (a) Microstructure of NDs (b) magnified [8]

Mechanical properties: The thermoplastic polymer nanocomposites showed high crystallintiy and high melting temperature when the filler concentration is increased and also the length of the polymer chain. When NDs are functionalized with hydroxyl groups and hydrogen bonds are formed with the matrix, fracture energy of PVA composites rise. When epoxy polymer was loaded with NDs, the glass transition temperature, hardness, and young's modulus all increased dramatically. From the micrograph of epoxy ND composite, we can see that the ND concentration is very high and the material showed major properties of ND and polymer acts as a binding medium for the nanoparticle [8].

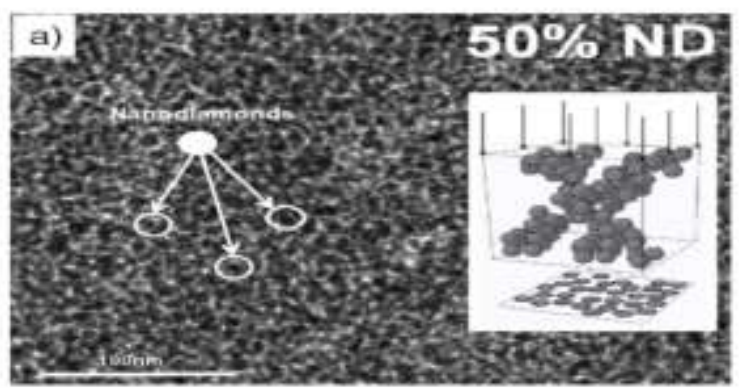

FIGURE 7. $50 \mathrm{wt} \%$ ND epoxy composite 3D structure [8]. 


\section{Electrospun Nanofibers}

The electrospinning process can be used to make a large range of nanofibers. The fibres have different applications in defence and security, biotechnology, structural monitoring, environmental engineering, and healthcare, reasons for applications are because of such unique properties some are described along the paper.

Electro Spinning Process: This is the most commonly used for the fabrication of long and continuous electrospun nanofibers. The method was developed by Anton Formals and patented in the year 1934[37]. The technique is mostly considered because of its low starting cost, simplicity, high production rate [35], and the different fiber morphologies and structures such as honeycomb, core-shell fiber, block copolymers, bead-on-a-string, side-by-side fibers, hollow, and yarn can be produced by controlling the process and solution parameters [37,19].

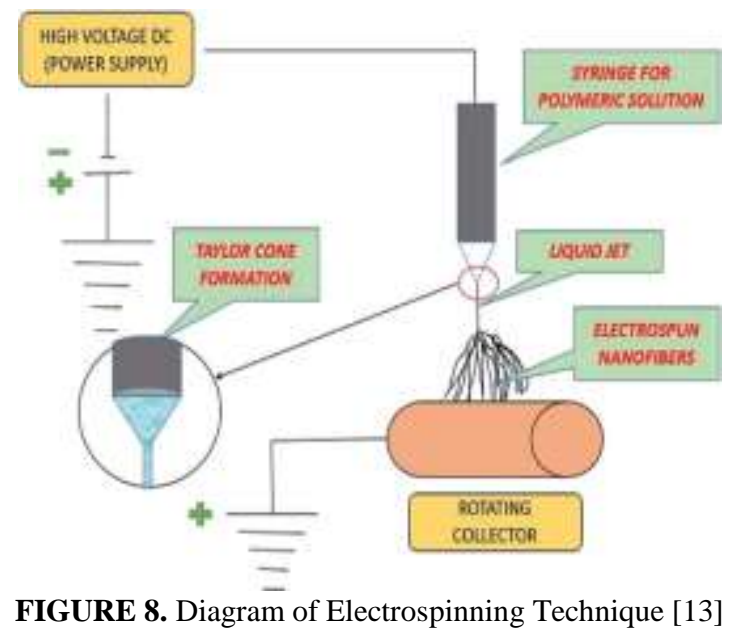

Working Principle: The following equipment is commonly used in the electro spinning process: A collector, a spinneret, a syringe, and a high voltage source (in $\mathrm{kV}$ ). A polymer dissolved in a volatile solvent, composite, sol-gel, or melts are put in the pipette or syringe during this process. At a set pace, the viscous polymer solution is pumped into the spinneret. To achieve adequate viscoelastic characteristics, the solvent concentrations are kept relatively high [35]. The particles in the solution are charged as a result of the high voltage delivered, resulting in electrostatic forces that operate as a repulsive force. The electrostatic forces overcome the surface tension when the voltage is raised. As a result, a jet is created. The positively charged solution exiting the metallic needle tip repels at the spinneret orifice, resulting in a charge accumulation at the tip of the metallic needle [19]. The jet is only stable at the spinneret in the electro spinning process, and owing to the interference between the electric field and the surface tension, the jet is prone to bending instability, causing the fibre to elongate. Many instabilities can induce breakage and hinder fibre development, but the entanglements in the polymer chains keep the jet from breaking apart, therefore bending instability is important in fibre production [38,39]. As the jet moves towards the lower potential region the solvent is evaporated and this causes the long and continuous fibers. The fibers are deposited and solidify on the collector which is oppositely charged $[19,38]$. The viscosity and surface tension should be adjusted appropriately to prevent the breaking of the varicose, if the varicose breaks up then electrospraying occurs this phenomenon is known as Rayleigh's Instability and usually occurs at low voltages and low concentrations, therefore it is important to apply high voltage $[19,39]$.

Electro Spinning Parameters: The Electrospinning parameters play a crucial role in controlling the fiber diameter, composition, orientation, and morphology. It is important to understand these parameters to determine the nanofibers structures produced, also it is not only important to understand their nature but also to understand the conversion of solutions or melts into nanofibers, and by controlling the parameters nanofibers with desired morphology can be fabricated. The electro spinning method is controlled by the following parameters:

1. Solution Parameters

2. Parameters of the process

3. Environmental/ambient parameters

Applied Voltage: The applied voltage has an impact on the fiber's length and diameter. It has been shown that a large potential difference can increase the fiber's length, and a higher voltage can cause the jet to travel faster towards the collector, reducing the time it takes for it to elongate before deposition, increasing the fiber's diameter [35-40]. To overcome surface tension and avoid electro spraying, a high voltage must be used [19].

Feed Rate: In the electro spinning technique, the feed rate has a significant impact on the influence on fibre diameter and morphology. Any variation from the ideal feeding rate of the solution or melts through the syringe pump will result in nanofibers with a wide width, and any divergence will result in a big fibre diameter [40]. If there is increase in rate of the solution, high fibers with large diameter are formed because the charge density decreases (when the charge density is high the fiber is subjected o secondary bending instabilities, therefore fibers with a small diameter are formed) which corresponds to the increase in nanofiber diameter and beads are also formed at high feed rate due to less time provided for solvent evaporation $[35,19]$. 
Collector type: Collector Distance is used to influence the orientation of the nanofibers, many collectors have been developed such as a Rotating drum, Rotating Disk, Rotating tube for controlling the alignment and orientation of nanofibers. Rotating drum collector is the most commonly used collector due to its simple setup and ability to fabricate a large area of highly aligned fibers. Uniaxially aligned fibres and highly aligned arrayed fibres are made with rotating disc collectors [35]. The drum and disk type collectors are preferred to plate type because they could fabricate highly aligned fibers, yarns braided, or random fibers [19].

Tip to Collector Distance: This may impact the fibre diameter; as the tip to collector distance rises, thin nanofibers are produced, which may cause rayleigh's instability, culminating in the formation of beads. Between the spinneret and the collector, a sufficient space should be maintained to allow for solvent evaporation and fibre elongation. [37, 19].

Viscosity: Viscosity plays a important role in the process. A polymer of high viscosity, the electrostatic force may not provide enough strength to stretch the melt to fabricate a nanofiber. Therefore, the concentration of the polymeric solution should be decreased but if it is decreased below a critical value then electro spraying occurs rather than electrospinning. Thus, it is very important to maintain enough viscosity in the solution so it can be stretched into a nanofiber; therefore entanglement number is used to determine the formation of the fiber.

\section{Properties of Electro spun Nanofibers}

Electro spun Nanofibers exhibit various properties in many different fields such as electricity, optics, thermotics, mechanics, chemistry, and magnetics. The electrospun nanofibers exhibit these numerous properties due to the surface, interface, and quantum size effect. The electrospun nanofibers are highly porous mesh and have a high S/V ratio (i.e., more surface area) and a web-like structure, therefore creating potential applications.

Porosity: Electrospun nanofibers are highly porous mesh, therefore, creating the application in energy storage and tissue engineering. The Porosity of the nanofiber mainly depends on two critical factors tip to collector distance and applied voltage. When the applied voltage has increased the porosity of the nanofiber is also increased and when the tip to collector distance has increased the porosity is decreased in the electro spun nanofiber. The porosity of the electrospun nanofiber is determined or measured by the density method or solution replacement method [37,41]. Due to the small pores present in the electrospun nanofibers, they can act as a storage device, and molecules can transfer between the pores of the nanofiber thus producing huge energy and storage capacity [37].

S/V Ratio: The surface area of electro spun nanofibers is large. Electro spun nanofibers can be utilized for energy conversion materials for energy devices because of their high S/V ratio, since the large surface area allows for quicker charge transfer and better ionic conductivity [37]. Usually, Electro spun nanofibers possessing a diameter in the range of 5 to $500 \mathrm{~nm}$ have a surface area of 10,000 to $1,00,000 \mathrm{~m}^{2} / \mathrm{kg}$, therefore the high surface area provides the excellent ability of the transfer of functional groups, ions and catalytic moieties thus they can be utilized as a separating material in the fuel cells and batteries [42].

\section{Applications of Electrospun Nanofibers}

Because of their exceptional characteristics, a wide variety of applications in the aerospace sector, biotechnology, healthcare, environmental engineering, and military and security. [35, 38]. Structural Health Monitoring System (SHM): Monitoring systems for structural health are used to detect, locate, characterize and quantify damage in simple and complex structures. The SHM can provide data about the health of the structure and assess the aircraft aging or damages thus reducing the maintenance cost and minimizing the scheduled periodic inspection, and improving safety and reliability [18]. Many smart materials have been developed for practical applications of SHM in infrastructure and they have been developed using nanotechnology as it could manipulate matter at the nanoscale and they can improve measurement and monitoring ability of the SHM at the nanoscale[44], and therefore Electrospun nanofibers are being used to manufacture the Structural health monitoring system as they have good mechanical properties they can easily resist the environmental/ambient conditions and due to their small size and less weight, they could be used in assessing the damages in aircraft, automobiles, etc. [18] Their large ratio and high porosity provide fast access to the analyte and a large signal-to-noise ratio; therefore, they are used to manufacture sensors [43]. PVDF-MWNCNT has been integrated into a GRPF to construct SHM's and has successfully been used to detect vibration, damping [3]. PAN-derived Carbon nanofibers are also used to manufacture SHM, they possess high tensile strength and high modulus of rupture, and large surface area which can be integrated with the structure [36, 44]. The PAN nanofibers have been shown to have higher strain sensing performance than any other alternative when undergone four circumferential probe measurements and thus making them a great strain sensor [44].

Self Healing Materials: Materials that can heal itself is a new class of smart nanomaterial applications with the potential to fully or partially heal and recover their original characteristics in the event of mechanical or thermal damage. The selfhealing materials promise an increase in the lifetime and enduring strength and these are very important in the materials where repair is not possible. Filled elastomers are used in solid propellant rockets and ballistic missiles, and it was demonstrated that mechanical activities induced nanoscale damage, which eventually turned into macroscopic fractures [45] and therefore causing loss to the material or the structure, and it was found that self-healing nanofibers can be used to prevent the damages. In the aircraft and aerospace industry, the damages within the structure are nearly impossible to detect, the electrospun core-shell nanofibers containing self-healing material that is a resin monomer inside the core of the nanofiber, and the catalyst is stored in the distribution in the matrix. When any pressure damage causes a crack, the catalyst 
contacts the monomer which initiates polymerization and thus self-heals damage [35]. Electrospun PAN nanofibers entangled mats consisting of resin monomer encased to form a composite were subjected to tensile testing have shown great self-healing properties and improved restoring of mechanical properties and therefore PAN-resin-cure nanofiber mats are being used for self-healing materials [46]. Self-healing PMMA has been used as fibre for electrospinning the core-shell nanofiber because it has a high modulus of rupture.

Sound Absorption Materials: In order to absorb high-frequency sound, sound-absorbing materials are employed. Sound absorption materials are very necessary to reduce noise pollution, commercial aircraft and drones use sound absorption materials to reduce the noise produced by their engines [17], therefore aircraft manufacturers are preparing new methods to manufacture the sound absorption materials to reduce the vibrations and sound produced by the engine and fuselage [35]. Porous Sound absorption materials are mostly used for sound absorption, these materials usually consist of small pores allowing sound waves to propagate in them, the waves are dissipated in the form of heat due to the friction in between the air molecules and pore walls. Therefore, Electro spun nanofibers are being used to prepare highly efficient noise absorption materials because of high porosity property and also because of their low starting cost, less weight, and large surface area offered by the electrospun nanofibers greater enhancement of dissipation of the sound waves. 3D nanofibers are utilised for sound absorption rather than 2D nanofibers because they include 95 percent porosity and so have a higher absorption. FIBER NFAs with a hierarchical pore structure with a main pore size of 10 to $30 \mathrm{~m}$ and a cell wall consisting of a tiny pore size ranging from 1 to $2 \mathrm{~m}$ are created to obtain an even greater sound absorption phenomenon. The FIBER NFAs possessed many connected microchannels where the sound waves are dissipated into heat energy due to viscous loss, therefore providing efficient sound absorption than any other commercial sound absorption material in the frequency range within 6300Hz [48]. Polymeric piezoelectric materials are fabricated for sound absorption as they can convert the mechanical energy into electrical energy, electro spun polyvinylidene fluoride (PVDF), and PVDF/graphene is an example of polymeric piezoelectric material and has been fabricated for sound absorption and has shown greater sound absorption in the middlefrequency range [49]. Electro spun silica fibres are also utilized for sound absorption, and in the range of frequencies between 1600 to $6400 \mathrm{~Hz}$, they outperform glass wool. The electro spun nanofibers are used as noise control material mostly in the aircraft as they occupy less space and due to their lightweight. If the thickness of electrospun nanofiber is increased the sound absorbance shifts towards low frequency and there is a decrease in absorption coefficient too, but if the fiber diameter increases beyond 500nm then the absorption coefficient remains constant but the sound absorbance shifts towards lower frequency [17]. The major application of NDs is in polymer composites due to its superior interaction and properties that have applications mainly in aerospace industry where it can be used as a structural material, for shielding, sensors and other electrical devices. The properties of ND reinforced polymers are found to be better than CNT and graphene-based composites. It is also having applications in energy storage electrical and environment as well [8, 34].

\section{Conclusion}

Nanotechnology, an emerging industry has attracted research enthusiasts worldwide. The nanomaterials with their ultrafine particle size exhibit amazing properties. Their properties include a high aspect ratio, thermal and electrical conductivity, and exceptional mechanical strength, all resulting from nanoscale size.

1) The various types of nanoparticles like metallic, carbon, and electron spun nanofibers with their properties open infinite applications.

2) The metallic nanoparticles are well known for their optical functionality useful in imaging and sensors.

3) Gold nanoparticles exhibit exceptional catalytic properties that are active even under mild conditions which can be used in fuel cells used as an energy source in spacecraft.

4) Silver nanoparticle, on the other hand, has excellent plasmon resonance and U-V is scattering in accordance with the Mie theory.

5) Aluminium nanoparticle has got lubricative property. It is wearing and heat resistant. SEM images of Al2O3 showed high thermal stability.

6) When it comes to carbon nanoparticles graphene and CNTs are the focus.

7) Nano diamonds with their high $\mathrm{S} / \mathrm{V}$ ratio are used in shielding and energy storage.

8) The electron spun nanofibers exhibit high fractural properties and impact energy. These particles mostly synthesized by electrospinning techniques have high porosity and surface area. These properties can be applied in the aerospace industry, environmental engineering, and health care.

To brief it out, nanoparticles whether metallic or carbon, all exhibit exceptional properties that are absent in their bulk materials. This very reason makes them more reliable. They are nowadays replacing bulk materials in many industries. Using these in the aerospace industry paves way for the production of lightweight cost-efficient aircraft with improved performance. This study describes the first effort to use the COOBL approach to improve the exploring capabilities of SKF. In addition, the suggested approach incorporates the leaping rate. If the jumping rate criterion is satisfied, the opposing solution is chosen if it is superior to the present one. The suggested COOBSKF is superior to SKF and better than GA, GWO, PSO, and BH, according to the study. Different OBL methods will be investigated in future studies to improve the SKF even further. 


\section{Reference}

[1]. Al Arsh Basheer, Advances in the smart materials applications in the aerospace industries, Aircraft Engineering and Aerospace Technology, 92 (7), 2020, 1027-1035.

[2]. Mutsumi Yoshida and Joerg Lahann, Smart Nanomaterials, ACS Nano, 2 (6), 2008, 1101-1107.

[3]. Stefania Gottardo, Agnieszka Mech, Jana Drbohlavová, Aleksandra Małyska, Søren Bøwadt, Juan Riego Sintes, Hubert Rauscher, Towards safe and sustainable innovation in nanotechnology: State-of-play for smart nanomaterials, NanoImpact, 21, 2021, 100297, 10, 1016.

[4]. P.Lakshmi and M.S.Nisha, Development of Smart Nano composites for Aerospace Structural Health Monitoring System, International Journal of Applied Engineering Research, 10(7), 16303-16320.

[5]. Jeffrey R. Potts, Daniel R. Dreyer, Christopher W. Bielawski, Rodney S. Ruoff, Graphene-based polymer nanocomposites, Polymer, 52(1), 2011, 5-25.

[6]. Zhenhao Li, Siyuan Chen, Shruti Nambiar, Yonghai Sun, Mingyu Zhang, Wanping Zheng and John T W Yeow, PMMA/MWCNT nanocomposite for proton radiation shielding applications, Nanotechnology, 27(23), 2016, 234001.

[7]. Velram Balaji Mohana, Kin-tak Laub, David Huic, Debes Bhattacharyyaa, Graphene-based materials and their composites: A review on production, applications and product limitations, Composites Part B, 142, 2018, $200-220$.

[8]. Vadym N. Mochalin, Yury Gogotsi, Nanodiamond-polymer composites, Diamond \& Related Materials, 58, 2015, $161-171$.

[9]. Ayesha Kausar, Amalgamation of Nanodiamond and Epoxy”, Kausar AAmerican Journal of Polymer Science \& Engineering, 5, 2017, 34-42.

[10]. R. Jose Varghese, El hadji Mamour Sakho, Sundararajan Parani, Sabu Thomas, Oluwatobi S. Oluwafemi and Jihuai $\mathrm{Wu}$, Introduction to nanomaterials: synthesis and Applications, Nanomaterials for solar cell applications, 7595, 2019.

[11]. Lev S. Novikov and Ekaterina N. Voronina, Potential Space Applications of Nanomaterials, J. Kleiman (ed.), Protection of Materials and Structures from the Space Environment, Astrophysics and Space Science Proceedings 47, 2017, 139-147.

[12]. Zishan H. Khan, Avshish Kumar, Samina Husain, and M. Husain, Introduction to Nanomaterials, chapter 1, Advanced Structured Materials, 2016, 1-23.

[13]. Harish Kumar K, Nagasamy Venkatesh*, Himangshu Bhowmik and Anuttam Kuila, (2018) "Metallic Nanoparticle: A Review, Biomedical Journal of Scientific \& Technical Research, Biomedical Research, 4(2), 2018, 3765-3775.

[14]. David T. Thompson, Using gold nanoparticles for catalysis, Nano Today, 2(4), 40-43.

[15]. Di Yan, Qian Tang2, Ahmed Kovacevic, Yuanxun Zhang, Wei Liu, Pinghua Liang2and Huijun Zhang, Designing nano-aluminum laden fuel pump for aviation applications, Proc IMechE Part E: J Process Mechanical Engineering $0(0), 2020,1-10$.

[16]. Prasad Govindrao Jamkhandea , Namrata W. Ghuleb , Abdul Haque Bamerc , Mohan G. Kalaskar, Metal nanoparticles synthesis: An overview on methods of preparation, advantages and disadvantages, and applications, Journal of Drug Delivery Science and Technology, 53, 2019, 101174.

[17]. Wee-Eong Teo, Potential Applications of Electrospun Fibers in Aviation Industry, 2016.

[18]. M.S. Nisha, Dalbir Singh, J.FreestaShiny, B. Sasirekha, Design and Manufacture of Nanofibers Using Electrospinning Technique for Aerospace Application, Applied Mechanics and Materials, 852, 2016, 72-78.

[19]. Muhammad Badmus, Jing Liu, Nü Wang, Norbert Radacsi, Yong Zhao, hierarchically electrospun nanofibers and their applications: A review, Nano Materials Science, 2020, doi.org/10.1016/j.nanoms.2020.11.003

[20]. Michael T Barako, Vincent Gambin, Jesse Tice, Integrated Nanomaterials for extreme thermal management: a perspective for aerospace applications, Nanotechnology, 29(15), 2018.

[21]. D Mailly, Nanofabrication techniques, The European Physical Journal Special Topics, 172, $2009,333-342$.

[22]. M. O. Stetsenko, S. P. Rudenko, L. S. Maksimenko, B. K. Serdega, O. Pluchery and S. V. Snegir, Optical Properties of Gold Nanoparticle Assemblies on a Glass Surface, Nanoscale Research Letters, 12(1), $2017,348$.

[23]. Huang, X., Jain, P. K., El-Sayed, I. H., \& El-Sayed, M. A, Gold nanoparticles: interesting optical properties and recent applications in cancer diagnostics and therapy, Nanomedicine, 2(5), 2007, 681-693.

[24]. A. Shenashen, Sherif A. El-Safty, and Emad A. Elshehy, Synthesis, Morphological Control, and Properties of Silver Nanoparticles in Potential Applications, Particle \& Particle Systems Characterization, 31(3), 2013, $293-316$.

[25]. F.R. Marciano, L.F. Bonetti, R.S. Pessoa, J.S. Marcuzzo, M. Massi, L.V. Santos, V.J. Trava-Airoldi, The improvement of DLC film lifetime using silver nanoparticles for use on space devices, Diamond \& Related Materials, 17, 2008, 1674-1679.

[26]. J. J. Mock, M. Barbic, D. R. Smith, D. A. Schultz, and S. Schultz, Shape effects in plasmon resonance of individual colloidal silver nanoparticle, Journal of chemical physics, 116(15), 2002, 6755. 
[27]. Muhammad Ilman Hakimi Chua Abdullah, Mohd Fadzli Bin Abdollah, Hilmi Amiruddin, Nur Rashid Mat Nuri, Noreffendy Tamaldin, Masjuki Hassan, S.A. Rafeq, Effect of hBN/Al2O3 nanoparticles on engine oil properties, Energy Education Science and Technology Part A: Energy Science and Research, 32(5), 2014, 3261-3268.

[28]. Ting Luo, Xiaowei Wein, Xiong Huang, Ling Huang, Fan Yang, Tribological properties of Al2O3 nanoparticles as lubricating oil additives, Ceramics International, 40, 2014, 7143-7149.

[29]. J Liu, B Cankurtaran, G McCredie, M J Ford, L Wieczorek and M B Cortie, Investigation of the optical properties of hollow aluminium 'nano-caps', Nanotechnology, 16, 2005, 3023-3028.

[30]. Lachebi, I., Fedala, A., Djenizian, T., Hadjersi, T., \& Kechouane, M, Morphological and optical properties of aluminum nanoparticles deposited by thermal evaporation on heated substrates, Surface and Coatings Technology, 343, 2018, 160-165.

[31]. Dilini Galpaya, Mingchao Wang, Meinan Liu, Nunzio Motta, Eric Waclawik, Cheng Yan, Recent advances in fabrication and characterization of graphene-polymer nanocomposites, Graphene, 1(2), 2012, 30-49.

[32]. Wenyan Liang, Fangxin Wang, Tong Earn Tay, Bin Yang, Zhenqing Wang, Experimental and Analytical Investigation of Epoxy/MWCNT Nanocomposites: Electrical, Thermal Properties, and Electric Heating Behavior, Polymer Engineering Science, 60, 2019, 233-242.

[33]. Suraj Rawal, James Brantley, Nafiz Karabudak, Development of Carbon Nanotube-based Composite for Spacecraft Components, RAST 2013 - Proceedings of 6th International Conference on Recent Advances in Space Technologies. 13-19. 10, 1109, 2013, 6581186.

[34]. Yinhang Zhanga, Kyong Yop Rheeb, David Huic, Soo-Jin Parka, A critical review of nanodiamond based nanocomposites: Synthesis, properties and applications, Composites Part B: Engineering, 143, 2018, 19-27.

[35]. Ahmed Barhoum, Kaushik Pal, Hubert Rahier, Hasan Uludag, Ick Soo Kim, Mikhael Bechelany, Nanofibers as new-generation materials: From spinning and nano-spinning fabrication techniques to emerging applications, Applied Materials Today, 17, 2019, 1-35.

[36]. Ibrahim M. Alarifi, Abdulaziz Alharbi, Waseem S. Khan, Andrew Swindle and Ramazan Asmatulu, Thermal, Electrical, and Surface Hydrophobic Properties of Electrospun PolyacrylonitrileNanofibers for Structural Health Monitoring, Materials, 8, 2015, 7017-7031.

[37]. Xiaomin Shi, Weiping Zhou, Delong Ma, Qian Ma, Denzel Bridges, Ying Ma, and Anming Hu, Electrospinning of Nanofibers and Their Applications for Energy Devices, Journal of Nanomaterials, 16(1), 2015.

[38]. Seeram Ramakrishna, Kazutoshi Fujihara, Wee-Eong Teo, Thomas Yong, Zuwei Ma, and Ramakrishna Ramaseshan, Electrospun Nanofibers Solving Global Issues, Materials Today, 9(3),2006, 40-50.

[39]. Farah Ejaz, Ahmed, Boor Singh Lalia, Raed Hashaikeh, A review on electrospinning for membrane fabrication: Challenges and applications, Elsevier, 356, 2015, 15-30.

[40]. Saikat Sinha Ray, Shiao-Shing Chen, Chi-Wang Li, Nguyen Cong Nguyen and Hau Thi Nguyen, A comprehensive review: electrospinning technique for fabrication and surface modification of membranes for water treatment application, RSC Advances, 6, 2016, 85495-85514.

[41]. Ting Wang, Wenxia Dong, Ying Chen2, Tiandi Pan, and Rudong Chen, Study on Porosity of Electrospun Nanofiber Membrane by Neural Network, Applied Mathematical Sciences, 12, 2018, 1059-1074.

[42]. Frank K. Ko, Heejae Yang, Functional Nanofibre: Enabling Material for The Next Generations Smart Textiles, Journal of Fiber Bioengineering and Informatics, 1(2), 2008, 81-92.

[43]. E. Zdraveva, J. Fang, B. Mijovic, T. Lin, Electrospun Nanofibers, Structure and Properties of High-Performance Fibers, 2017, 267-300.

[44]. Ibrahim M. Alarifi, Abdulaziz Alharbi, Waseem S. Khan, and Ramazan Asmatulu, Structural Health Monitoring of Composite Aircraft, Advances in Materials Science Research, 2015, 268-300.

[45]. Richard P. Wool, Self-healing materials: a review". Soft Matter, 4(3), 2008, 400-418.

[46]. Min Wook Lee, Seongpil An, Hong Seok Jo, Sam S. Yoon, Alexander L. Yarin, Self-healing NanofiberReinforced Polymer Composites: Tensile Testing and Recovery of Mechanical Properties, ACS Applied Materials \& Interfaces, 7(35), 2015, 19546-19554.

[47]. Rasoul Esmaeely Neisiany, Jeremy Kong Yoong Lee, Saied Nouri Khorasania, Rouhollah Bagheri, and Seeram Ramakrishna, Facile strategy toward fabrication of highly responsive self-healing carbon/epoxy composites via incorporation of healing agents encapsulated in poly(methylmethacrylate) nanofiber shell, Journal of Industrial and Engineering Chemistry, 59, 2018, 456-466.

[48]. Leitao Cao, Qiuxia Fu, Yang Si, Bin Ding, Jianyong Yu, Porous materials for sound absorption, Elsevier, 10, 2018, 25-35.

[49]. Chang Mou Wu, Min Hui Chou, Sound Absorption of Electrospun Polyvinylidene Fluoride/Graphene Membranes, European Polymer Journal, 82, 2016, 35-45. 